\title{
Saffron (Crocus Sativus L.), Combined with Endurance Exercise, Synergistically Enhances BDNF, Serotonin, and NT-3 in Wistar Rats
}

\author{
Maryam Akbari-Fakhrabadi*1, Mohammad Najafi ${ }^{2}$, Soudabehsadat \\ Mortazavian ${ }^{1}$, Amir-Hossein Memari ${ }^{1}$, Farzad Shidfar ${ }^{3}$, \\ Ali Shahbazi ${ }^{4,5}$, Javad Heshmati ${ }^{6}$
}

\begin{abstract}
Background: Evidence indicates that combined approaches based on exercise and nutrition benefit neural development. We aimed to determine the effect of saffron and endurance training on hippocampus neurogenic factors, neurotrophin-3 gene expression in soleus muscle, and short-term memory in Wistar rats.

Methods: The study analyzed four groups of ten rats each: control, exercise, saffron, and saffron plus exercise. The rats in the exercise groups were trained on a rodent motor-driven treadmill. All rats were gavage daily with either saffron extract (40 mg/kg) or water. After eight weeks of intervention all rats were evaluated using the novel object recognition (NOR) test. Blood and tissue samples were collected to measure proteins and neurotrophin-3 gene expression.

Results: Rats that received saffron treatment combined with exercise had significantly greater brain-derived neurotrophic factor $(B D N F)$ and serotonin in hippocampus compared to the control and saffron-only-treated rats ( $\mathrm{p}$ 0.05). Neurotrophin-3 mRNA in soleus muscle was higher in the saffron plus exercise group than rats in the other three groups $(\mathrm{p}<0.05)$. Hippocampus 5-hydroxyindolacetic acid and short-term memory were significantly greater in all the intervention groups than in the control group $(\mathrm{p}<0.05)$.

Conclusions: Saffron, combined with endurance exercise, synergistically increased hippocampus BDNF, serotonin, and muscular neurotrophin-3 mRNA in Wistar rats.
\end{abstract}

Keywords: Endurance Exercise, Memory, Hippocampus, Saffron, Neurotransmitter.

\section{Introduction}

Studies have revealed that exercise improves neurogenesis and cognitive function (1). Neurogenesis has been linked to exercise through up-regulation of neurotrophins to a great extent in the hippocampal denate gyrus. The hippocampus is a vital region for memory consolidation and learning (2). Therefore, new-born neurons integrate into the hippocampal circuitry and consequently benefit hippocampal-dependent learning and memory tasks (3).
One important neurotrophin is brain-derived neurotrophic factor $(B D N F)$, which appears to be regulated by physical activity. This factor is a member of the nerve growth factors family with about $70-80 \%$ of circulating $B D N F$ secreted from the brain (4). Research has shown aerobic exercise elevates $B D N F$ gene $(B D N F)$ expression in the hippocampus, which results in dendritic complexity, synaptic plasticity, and enhanced memory and learning (5). 
Serotonin (5-hydroxytryptamine; 5-HT) has also been shown to play a critical regulatory role in hippocampal neurogenesis (6). Accumulating evidence indicates that serotonin production and metabolism during or after exercise increase in various regions of the brain including hippocampus, amygdala, basal ganglia, and cortex, which in turn ameliorates depression (7, 8). Further studies have shown that the denate gyrus is rich in serotonergic fibers which, in response to exercise, modulate neurogenesis (6). Insulin-like growth factor-1 (IGF-1) is another neurotrophic factor that has been shown to regulate adult brain neurogenesis (9). Exercise increases the availability of this growth factor, which enhances neurogenesis and improves brain function (10). $B D N F$, serotonin, and IGF-1 have been shown to activate similar signaling cascades that may act synergistically in neuro-homeostasis and neurogenesis (11).

Moreover, evidence indicates that combined approaches based on exercise and nutritional management can benefit neural development. Brain function undoubtedly requires adequate nutrition. Thus, variations in the composition and quantity of nutrient consumption likely influence cognitive function. Nutricuetical factors provide the essential material for the brain to create and maintain connections critical for neural plasticity and brain function (12). Studies show growing interest in the potential beneficial effects of polyphenols on brain health. Polyphenols, which have strong antioxidant properties, are micronutrients in plant-originated food. Food and beverages including cocoa, coffee, brightlycolored fruits and vegetables, and tea are the major dietary sources of polyphenols. It has been suggested that their neuro-protective effects occur via reducing neuro-inflammation and they enhance cognitive function and promote memory and learning (13).

Crocus sativus L., commonly known as saffron, is a member of the Iridaceae family, which is rich in carotenoids including crocin, crocetin, safranal, and picrocrocin, and flavonoids including quercetin, kaempferol, and naringenin $(14,15)$. In traditional medicine, saffron has been used to treat fever and asthma, and as an analgesic, expectorant, sedative, and mood stabilizer (16). Recent pharmacological studies have shown that saffron increased related brain neurotransmitters in animal models of depression (17) and the possible mechanism has been shown to occur via $B D N F$ and $V G F$ neuropeptide upregulation in the hippocampus (18). However, due to the limitations in human trials, it is not yet known whether saffron can directly induce neurodevelopmental biomarkers to enhance memory. Thus, by relying on the positive effects of exercise on neurogenesis and memory we aimed to determine the effect of saffron and endurance training individually and combined on hippocampus neurogenic factors and short-term memory in Wistar rats.

We also aimed to evaluate the effect of saffron combined with exercise on neurotrophin-3 (NT3) mRNA level in soleus muscle. NT-3 is an important autocrine neurotrophic factor that stimulates differentiation and neuron outgrowth in central and peripheral nerves in target tissues such as skeletal muscle (19). Studies have shown that voluntary exercise in normal rate increases NT-3 mRNA expression in soleus muscle (20); however, the role of saffron in inducing this neurotrophic factor alone or synergistically with exercise has not yet been studied.

\section{Materials and Methods \\ Saffron extract preparation}

The saffron used in this study was provided by Saharkhiz Co. (Mashhad, Iran). Its herbarium code is 829-537-06. A hydroalcoholic form was prepared by percolation. Briefly, in an ultrasonic bath each gram of the air-dried stigma was extracted with $33 \mathrm{ml}$ of an $80 \%$ ethanol solution. The extract was filtered, isolated on a percolation rotary evaporator, and stored at $-20{ }^{\circ} \mathrm{C}$ until use. The dosage of saffron extract was $40 \mathrm{mg} / \mathrm{kg}$ which was dissolved in $1.5 \mathrm{ml}$ water according to a previous study (21).

\section{Animals}

Forty male Wistar rats with a mean weight of 198 $\pm 8.20 \mathrm{~g}$ at the beginning of the study were provided by the animal house of Iran University of Medical Sciences and 10 rats were randomly allocated to each of four groups: Exercise, Saffron, Exercise plus Saffron, and Control. The animals 
were maintained in stainless cages with four animals per cage and kept in a standard atmosphere. The room temperature was $22 \pm 2$ ${ }^{\circ} \mathrm{C}$, humidity was $50 \pm 5 \%$ with a twelve-hour light-dark cycle (8:00-20:00). Food (pellet) and water (specified water bottles) were available at all times. The rats were acclimated to the location for one week prior to intervention. All animals were gavaged with either water as control or saffron extract (40 $\mathrm{mg} / \mathrm{kg}$ ) with the same volume for eight weeks (21). The animals were weighed weekly to maintain the intervention dosage during the trial. All animal experimental approaches were proved and applied by the Animal Care and Use Committee (ACUC) at IUMS, which was in accordance with the NIH guidelines (22). The ethical code registered for this study was IR.IUMS.FMD.REC1396.9223475203.

\section{Endurance training}

Endurance training was performed on a treadmill specified for rats. The Exercise and Exercise + Saffron groups were acclimatized for three days on the treadmill for 10 minutes at a speed of 8 $\mathrm{m} / \mathrm{min}$. The exercise was performed five days per week (Saturday through Wednesday) at 11:00-13:00 for eight weeks. During the eight weeks the exercise time and intensity were increased progressively. Sixty percent of $\mathrm{VO}_{2} \mathrm{Max}$ was considered as the initial speed of training. This speed was calculated by a gradually increasing ramp protocol. Accordingly, the starting running speed was 8 $\mathrm{m} / \mathrm{min}$, then the speed was increased every two $\min$ by $2 \mathrm{~m} / \mathrm{min}$. Finally, $\mathrm{VO}_{2} \mathrm{Max}$ was considered at the speed the rats were unable to maintain even by mild taping on their tails for one min. The mean $\mathrm{VO}_{2} \mathrm{Max}$ was computed at approximately $20 \mathrm{~m} / \mathrm{min}$. According to the $\mathrm{VO}_{2} \mathrm{Max}$, the training regimen was designed in this way: first week: $12 \mathrm{~m} / \mathrm{min}, 20 \mathrm{~min}$; second week: $12 \mathrm{~m} / \mathrm{min}, 30 \mathrm{~min}$; third week: $15 \mathrm{~m} / \mathrm{min}$, $30 \mathrm{~min}$; fourth week: $15 \mathrm{~m} / \mathrm{min}, 35 \mathrm{~min}$; fifth week: $18 \mathrm{~m} / \mathrm{min}, 35 \mathrm{~min}$; sixth week: $18 \mathrm{~m} / \mathrm{min}$, $40 \mathrm{~min}$; seventh week: $20 \mathrm{~m} / \mathrm{min}, 40 \mathrm{~min}$; eighth week: $20 \mathrm{~m} / \mathrm{min}, 45 \mathrm{~min}$. The rats were warmed up for five min at $8 \mathrm{~m} / \mathrm{min}$ before each exercise session. A hard sponge was placed at the end part of the treadmill with no shocker bars connected. In addition, to limit stress on the rats, which would adversely affect the results, the rats' tails were gently tapped.

\section{Blood and tissue sample collection}

To prevent the metabolic effects of the final run, $48 \mathrm{~h}$ after the last exercise session all animals were anaesthetized with xylazine (10 $\mathrm{mg} / \mathrm{kg}$ ) and ketamine (120 mg/kg). Next, blood was collected via cardiac puncture, plasma was extracted, and samples were stored at $-80{ }^{\circ} \mathrm{C}$. Then, all the animals were euthanized through spinal dislocation to limit suffering immediately after blood collection. Finally, in less than four min, the soleus muscles from both legs and hippocampus from both sides of the brain were dissected, frozen in liquid nitrogen, and stored at $-80{ }^{\circ} \mathrm{C}$ for further protein and genetic analysis.

\section{BDNF, IGF-1, Serotonin, 5-HIAA, 5HIAA/serotonin}

Both sides of the hippocampus from each rat were minced to small pieces, rinsed in ice-cold PBS (0.01 M, pH=7.4) to remove excess blood, and chopped into $1-2 \mathrm{~mm}$ pieces. Then they were homogenized using a glass homogenizer in lysis buffer containing $50 \mathrm{mM}$ Tris- $\mathrm{HCl}(\mathrm{pH} 8.0)$, $150 \mathrm{mM} \mathrm{NaCl}, 10 \%$ glycerol, $1 \%$ Triton X-100, $1.5 \mathrm{mM} \mathrm{MgCl} 2 \cdot 6 \mathrm{H} 2 \mathrm{O}, 1 \mathrm{mM}$ EGTA, $1 \mathrm{mM}$ PMSF, $1 \mathrm{mM} \mathrm{Na2VO4,} \mathrm{and} 100 \mathrm{mM} \mathrm{NaF}$, centrifuged at $3300 \mathrm{RCF}$ for $20 \mathrm{~min}$, and the supernatant was removed for the ELISAs. Hippocampal and plasma $B D N F$ were measured by the BDNF ELISA Kit (MyBioSource Inc. Cat. No: MBS824814, California, San Diego, USA). IGF-1 was assessed by ELISA Kit (Cusabio Technology LLC. Cat. No: CSBE04582r, Houston, San Diego, USA). Serotonin was measured by the Serotonin ELISA Kit (MyBioSource Inc. Cat. No: MBS9362408, California, San Diego, USA). 5hydroxyindolacetic acid (5-HIAA) was measured by ELISA Kit (MyBioSource Inc. Cat No: MBS700811, California, San Diego, USA). The 5HIAA/serotonin ratio was determined and considered as an index of neurotransmitter metabolism. 


\section{Isolation of RNA and qRT PCR}

Rat soleus muscle total RNA was extracted using a BioFact HiGene RNA Prep Kit (Cat. No. RP101-050, Korea). The quality and quantity of the isolated RNA were determined on a Nanodrop spectrophotometer. The samples were stored at $80{ }^{\circ} \mathrm{C}$ until use. A Takara Kit (Cat. No. 6110A, Japan) was used for cDNA synthesis. NT-3 mRNA expression was assessed by comparing its amplification against $\beta$-actin. The primers used in this study were: NT-3 Forward:

5'-CAAAGCAGAGGCACCCAGAGAACC-3', NT-3 Reverse:

\section{5'GTGTCTATTCGTATCCAGCGCCAGC-3',}

$\beta$-actin Forward:

5'GCCAACCGTGAAAAGATGAC-3',

$\beta$-actin Reverse:

5'AGCGCGTAACCCTCATAGAT-3'.

The mRNA expression was evaluated by PCR Master Mix (Takara, Japan). Expression for all samples was measured twice. Each sample included $0.5 \mu \mathrm{L}$ of each primer, $1 \mu \mathrm{L}$ of the DNA, $7.5 \mu \mathrm{L}$ of SYBR Green, $0.3 \mu \mathrm{L}$ of Rox, and 5.2 $\mu \mathrm{L}$ of RNasefree water. An ABI PRISM (7700 Sequence Detection System, Applied Biosystems) was used for the Real-time PCR through the following procedure: 2 min of heating at $94^{\circ} \mathrm{C}$ followed by 45 cycles of denaturation at $94^{\circ} \mathrm{C}$ for $30 \mathrm{sec}$ and annealing and extension at $61^{\circ} \mathrm{C}$ for $30 \mathrm{sec}$.

\section{Novel object recognition test}

The hippocampus is an important brain region for spatial and recognition memory (23). Therefore, we evaluated the effect of saffron and endurance training either individually or combined on the rats' short-term memories by the novel object recognition (NOR) test. This test is based on the rat's natural exploratory behavior. On day one, the rats were habituated to the testing box for one $\mathrm{h}$ with no objects. After 24 hours, they were exposed for three minutes to two identical objects set in opposite corners of the testing box. One $h$ later, they were exposed to one familiar object as before and one new object placed in the corners of the box as before for three minutes (Fig. 2A). In the current study, "exploration" was defined as the movement of the snout towards the object, or touching or sniffing with the snout. Sitting on the object, climbing on, or running around it, were not considered exploration. The novel and familiar objects differed in color, size, and shape, which contributed to recognizing the novel object. After each testing session the box and objects where cleaned with $10 \%$ ethanol to remove odor cues. To analyze the test, we used the Discrimination Index (DI), which is used to differentiate the exploration time between the familiar and novel objects (24).

DI $=[($ Novel Object Exploration Time - Familiar Object Exploration Time)/Total Exploration Time] $\times 100$

\section{Statistics}

The data was analyzed using SPSS 24 (IBM, Chicago, IL, USA). The Shapiro-Wilk test was used to evaluate the normal distribution of the data. ANOVA and Post-Hoc LSD test were used to analyze normally-distributed data. The MannWhitney U-test was used to analyze the genomic factor NT-3. The threshold cycles (Cts) attained for NT-3 and $\beta$-actin were used for the genomic analysis. Quantitative gene expression was obtained by measuring $2^{-\Delta \Lambda \mathrm{Ct}}$ for each sample $(\Delta \mathrm{Ct}$ $\left.=\mathrm{Ct}_{N T 3}-\mathrm{Ct}_{\beta \text { Actin }}\right)$. In this study $\mathrm{P}$ values less than 0.05 were considered statistically significant.

\section{Results \\ Saffron analysis}

The total hydroalcoholic extract of saffron was $56 \%$. The flavonoid content of the extract considered as equivalent to rutin was $65.41 \pm 0.89$ $\mathrm{mg} / \mathrm{g}$, and the phenol content considered as equivalent to gallic acid was $60.07 \pm 1.90 \mathrm{mg} / \mathrm{g}$.

\section{BDNF, IGF-1, Serotonin, 5HIAA, 5HIAA/Serotonin}

Table 1 shows the brain biomarker concentrations after the various interventions. Plasma $B D N F$ concentration of Exercise + Saffron group was significantly greater than those of the other groups. $B D N F$ in hippocampus was significantly higher in the Exercise + Saffron group compared to Control and Saffron treated rats. No significant difference in IGF-1 concentration was detected between the groups. The serotonin concentration was significantly greater in the Exercise + Saffron group than in the Control and Saffron treated 
groups. 5HIAA was significantly greater in the Exercise, Saffron, and Exercise + Saffron groups than in the Control group. The 5HIAA/serotonin ratio was significantly greater in the Exercise and Saffron groups than in the Control group; however, no significant difference in this ratio was seen between the Exercise + Saffron and the other groups.

Table 1. Concentrations of BDNF, IGF-1, Serotonin, 5HIAA and 5HIAA/Serotonin ratio after intervention

\begin{tabular}{|c|c|c|c|c|c|c|c|c|}
\hline Variable & $\begin{array}{l}\text { Control } \\
(n=10)\end{array}$ & $\begin{array}{l}\text { Exercise } \\
(n=10)\end{array}$ & $\begin{array}{l}\text { Saffron } \\
(\mathrm{n}=10)\end{array}$ & $\begin{array}{l}\text { Exercise + } \\
\text { Saffron } \\
(\mathbf{n}=10)\end{array}$ & $p$ value & df & $\begin{array}{l}\text { Mean } \\
\text { square }\end{array}$ & $\mathbf{F}$ \\
\hline $\begin{array}{l}\text { Tissue BDNF } \\
\text { (pg/mg - } \\
\text { protein) }\end{array}$ & $4.35 \pm 0.32$ & $4.55 \pm 0.37$ & $4.50 \pm 0.44$ & $4.84 \pm 0.19^{\mathrm{acc}}$ & $0.024^{*}$ & 3 & 0.42 & 3.53 \\
\hline $\begin{array}{l}\text { Plasma BDNF } \\
(\mathrm{pg} / \mathrm{ml})\end{array}$ & $9.30 \pm 0.52$ & $9.35 \pm 0.26$ & $9.51 \pm 0.31$ & $10.00 \pm 0.50^{\mathrm{ab}, \mathrm{c}}$ & $0.002^{*}$ & 3 & 1.03 & 5.94 \\
\hline $\begin{array}{l}\text { Tissue IGF-1 } \\
\text { (pg/mg-tissue) }\end{array}$ & $20.64 \pm 0.43$ & $20.80 \pm 0.82$ & $20.68 \pm 1.21$ & $21.01 \pm 0.82$ & 0.77 & 3 & 0.27 & 0.36 \\
\hline $\begin{array}{l}\text { Serotonin } \\
\text { (ng/mg- } \\
\text { protein) }\end{array}$ & $15.54 \pm 0.61$ & $15.94 \pm 0.27$ & $15.72 \pm 0.44$ & $16.28 \pm 0.38^{\mathrm{ac}}$ & $0.005^{*}$ & 3 & 1.02 & 5.12 \\
\hline $\begin{array}{l}\text { 5HIAA } \\
\text { (ng/g-tissue) }\end{array}$ & $83.85 \pm 1.91$ & $89.73 \pm 3.31^{\mathrm{a}}$ & $89.97 \pm 1.94^{\mathrm{a}}$ & $90.92 \pm 2.01^{\mathrm{a}}$ & $<0.001^{*}$ & 3 & 103.66 & $\begin{array}{l}18.4 \\
2\end{array}$ \\
\hline $\begin{array}{l}\text { 5HIAA/ } \\
\text { Serotonin }\end{array}$ & $5.39 \pm 0.17$ & $5.63 \pm 0.26^{\mathrm{a}}$ & $5.72 \pm 0.20^{\mathrm{a}}$ & $5.58 \pm 0.19$ & $0.012^{*}$ & 3 & 0.189 & 4.19 \\
\hline
\end{tabular}

Data represented as Mean \pm SD

$* \mathrm{p}<0.05$ representative of a significant level by comparing between-group differences using ANOVA.

Significant $\mathrm{p}$ value by comparing within-group differences using Post-hoc LSD test presented as a: compared to the Control group; b: compared to the Exercised group c: compared to the saffron-treated group.

\section{Real-time q-PCR}

The Kruskal-Wallis test indicated a significant difference in NT-3 mRNA expression between four groups $(\mathrm{p}<0.001)$. According to the MannWhitney U test both the Exercise and Saffron

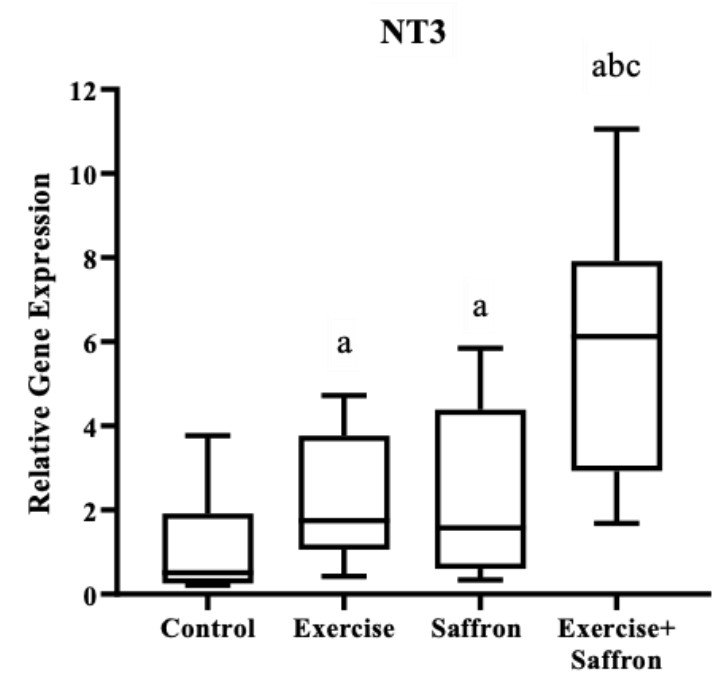

groups expressed significantly more NT-3 mRNA than the Control group, while the Exercise + Saffron group expressed significantly more NT-3 mRNA than all the other groups ( $\mathrm{p}<0.01$ ) (Fig. 1).

Fig. 1. Relative NT-3 mRNA expression in soleus muscle from four experimental groups. Letters represent a significant difference $(\mathrm{p}<0.05)$ compared to a: Control group, $b$ : Exercised group, and c: Saffron-treated group (MannWhitney U-test analysis). The solid lines across the boxes represent the medians. 


\section{Novel object recognition test}

All rats were habituated to the atmosphere of the novel object box for one $h$ one day before the main test. In the main test two rats from the Control group, one from the Exercise group, two from the Saffron group, and one from the Exercise + Saffron group were excluded due to no exploring movements. The rats in the three treatment groups spent more time than the Control group exploring the novel vs. the familiar object (Fig. 2B). The discrimination index shows this difference was statistically significant (Fig. 2C).
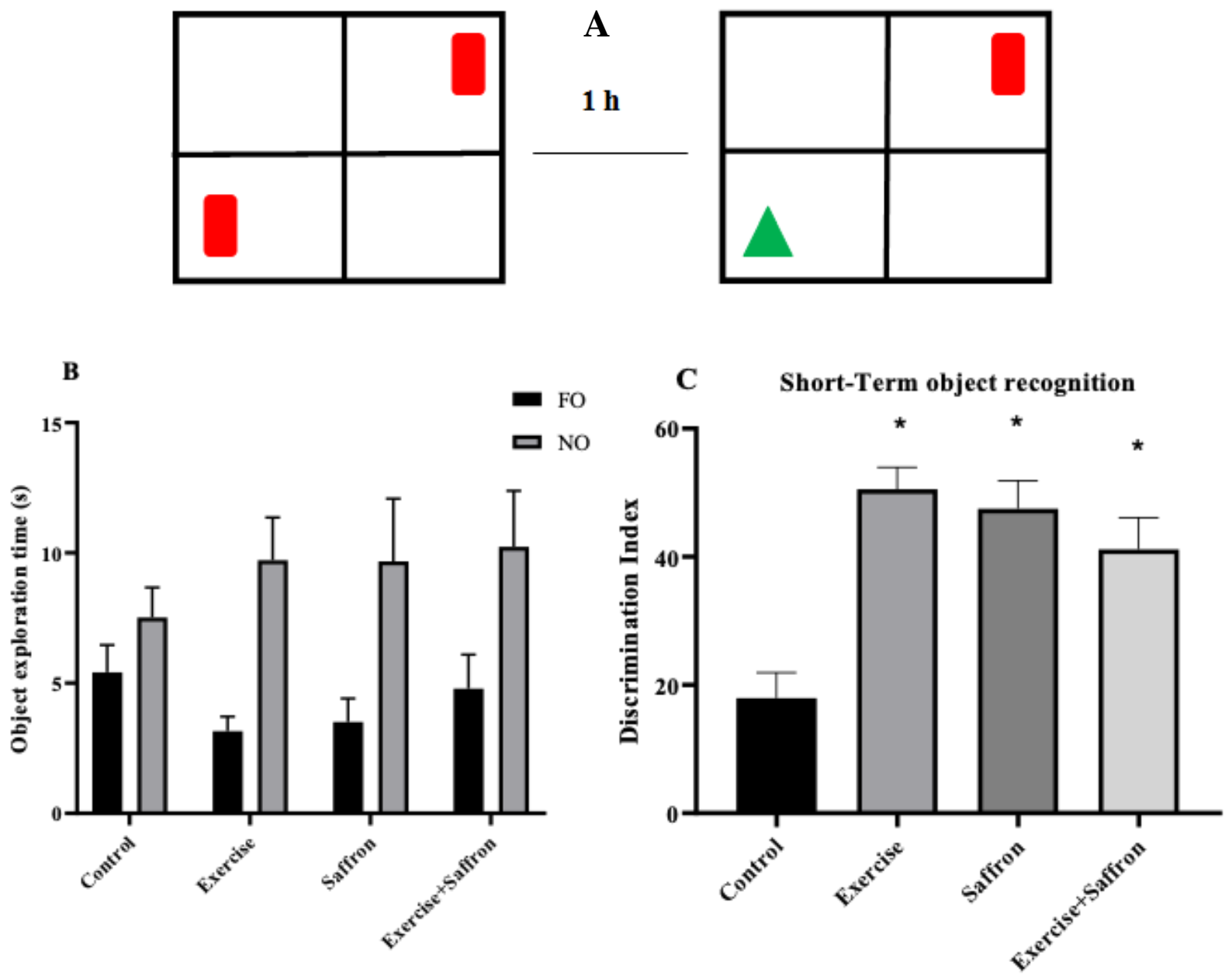

Fig. 2. Novel object recognition test: (A) Schematic illustration of the object recognition test. Rats were first exposed to an identical pair of objects. After $1 \mathrm{~h}$, they were exposed to the familiar object (FO) and a novel object (NO). (B) Time (s) spent exploring the familiar and novel objects in the second part of the test. (C) The Exercise, Saffron, and Exercise + Saffron groups all spent significantly more time on the novel object than the familiar object compared with the Control group, as determined by the discrimination index. Bars represent means (SEM). * $\mathrm{p}<0.05$.

\section{Discussion}

In this study saffron combined with endurance training increased $B D N F$ protein concentration in hippocampus and plasma. Previous studies mainly studied saffron anti-depressant effect, emphasizing its role in BDNF elevation (18). Because $B D N F$ plays an important role in augmenting synaptic plasticity and consequently improving memory (25), we further assessed the effect of the interventions on rat short-term memory using the NOR test. We found that Saffron, Exercise, and Exercise + Saffron all improved performance on the test. Previous studies showed that aerobic training increases $B D N F$ and consequently improves spatial memory (26-28). However, the effect of saffron on spatial memory has been less studied. Crocin 
has been reported to improve cognition and memory in Alzheimer's disease-induced mice (29) and also improve rat memory and learning (30). The cellular and molecular action of BDNF on neurogenesis and consequently on memory is initiated by the tropomyosin-related kinase B (TrKB) receptor, a member of the tyrosine kinase receptor family. The interface between $B D N F$ and the receptor results in tyrosine phosphorylation in the tyrosine kinase domain, and subsequent adaptor protein connection. Finally, these adaptors trigger the ras/mitogen-activated protein kinase (Ras-MAPK) pathway, resulting in neuronal proliferation and differentiation (32).

In our study, hippocampal IGF-1 was greater in the Exercise + Saffron group than in the other groups, but the difference was not significant. Similarly, Gaeini et al. showed that saffron combined with resistance training for six weeks had no effect on IGF-1 serum levels (33); however, evidence is lacking on the effect of saffron on hippocampal IGF-1 levels. A strong association has been shown between musculoskeletal mass and IGF-1; therefore, muscle enlargement during resistance training could increase IGF-1 levels (33); in contrast, in our study aerobic training was employed.

In our study, serotonin concentration in the hippocampus was greater in the Exercise + Saffron group compared to Control and saffron treated rats. During physical exercise serotonin is released from projections in the hippocampus, which affects serotonin receptors and triggers the onset of brain neurogenesis (6). Further, serotonin is a neurotransmitter involved in maintaining memory (34). However, in the Exercise and saffron-only-treated groups, serotonin showed no significant increase even though the rats improved significantly in the NOR test; this could be due to the high metabolism of serotonin. In addition, serotonin metabolism (5HIAA/serotonin) increased in the exercisetrained and saffron-treated groups. Because serotonin is an unstable neurotransmitter catabolized by monoamine oxidases (MAOs) to its metabolite, it is interpreted that saffron and exercise may have increased the main pool of serotonin individually, but its metabolism prevented its maintenance. However, in the
Exercise + Saffron group, the main pool of serotonin was significantly greater, while serotonin metabolism was less. This could be due to the potency of saffron to inhibit serotonin reuptake and maintain it at high levels in the brain (35). In light of the positive effects of saffron and exercise on memory in this study, it seems that one possible mechanism could be mediated by $B D N F$ and serotonin-stimulating neurogenic effects.

Our study is the first to evaluate the effect of saffron alone or combined with exercise on NT-3 in soleus muscle. We found that while both saffron and training increased NT-3 mRNA expression individually, when combined, this effect was even greater. NT-3 is produced by neurons, which play an essential role in the plasticity of the central nervous system and regeneration in spinal cord and muscle (19). Exercise could increase expression of NT-3 and its receptor, tyrosine kinase $\mathrm{C}$ (Trk $\mathrm{C}$ ) in soleus muscle (20). Sharma et al. reported that moderateintensity aerobic training induced NT-3 in skeletal muscle and also reduced muscle pain (36). However, no study has yet evaluated the effect of saffron on NT-3. The antioxidant and antiinflammatory properties of saffron may have prevented muscle damage due to exercise (37) and provided an appropriate medium for the regeneration of the neuromuscular region.

This study showed that saffron extract combined with endurance exercise increases hippocampus and plasma $B D N F$, hippocampus serotonin, and muscular NT-3 gene expression. All three interventions increased hippocampus 5HIAA and improved NOR test results compared to control. However, no significant effect was observed on hippocampal IGF-1 in this study.

Our study has limitations as follows: this was an experimental study accomplished in rats; results may differ in humans, and limited neurotrophins were evaluated in the tissues, which could be expanded in future studies.

\section{Acknowledgements}

We express our sincere gratitude towards all the laboratory specialists who supported this project. The authors declare that they have no conflicts of interest. 


\section{References}

1. Cotman CW, Berchtold NC, Christie LA. Exercise builds brain health: key roles of growth factor cascades and inflammation. Trends Neurosci. 2007;30(9):464-72.

2. Liu PZ, Nusslock R. Exercise-mediated neurogenesis in the hippocampus via $B D N F$. Front Neurosci. 2018;12:52.

3. Christian KM, Song H, Ming G-l. Functions and dysfunctions of adult hippocampal neurogenesis. Annu Rev Neurosci. 2014;37:243-62.

4. Rasmussen P, Brassard P, Adser H, Pedersen MV, Leick L, Hart E, et al. Evidence for a release of brain-derived neurotrophic factor from the brain during exercise. Exp Physiol. 2009;94(10):1062-9. 5. Hall JM, Gomez-Pinilla F, Savage LM. Nerve Growth Factor Is Responsible for ExerciseInduced Recovery of Septohippocampal Cholinergic Structure and Function. Front Neurosci. 2018;12:773.

6. Klempin F, Beis D, Mosienko V, Kempermann G, Bader M, Alenina N. Serotonin is required for exercise-induced adult hippocampal neurogenesis. J Neurosci. 2013;33(19):8270-5.

7. Rethorst CD, Wipfli BM, Landers DM. The antidepressive effects of exercise: a meta-analysis of randomized trials. Sports Med. 2009;39(6):491-511.

8. Ernst C, Olson AK, Pinel JP, Lam RW, Christie BR. Antidepressant effects of exercise: evidence for an adult-neurogenesis hypothesis?. J Psychiatry Neurosci. 2006;31(2):84-92.

9. Mir S, Cai W, Carlson SW, Saatman KE, Andres DA. IGF-1 mediated neurogenesis involves a novel RIT1/Akt/Sox2 cascade. Scientific reports. 2017;7(1):3283.

10. Huang T, Larsen KT, Ried-Larsen M, Moller NC, Andersen LB. The effects of physical activity and exercise on brain-derived neurotrophic factor in healthy humans: A review. Scand J Med Sci Sports. 2014;24(1):1-10.

11. Fidalgo S, Ivanov DK, Wood SH. Serotonin: from top to bottom. Biogerontology. 2013;14(1):21-45.

12. Meeusen R. Exercise, nutrition and the brain. Sports Med. 2014;44 Suppl 1(Suppl 1):S47-56.

13. Vauzour D. Dietary polyphenols as Hmodulators of brain functions: biological actions and molecular mechanisms underpinning their beneficial effects. Oxid Med Cell Longev. 2012;2012:914273.

14. Bolhassani A, Khavari A, Bathaie SZ. Saffron and natural carotenoids: Biochemical activities and anti-tumor effects. Biochim Biophys Acta. 2014;1845(1):20-30.

15. Varasteh A-R, Sankian M, Midoro-Horiuti T, Moghadam M, Shakeri MT, Brooks EG, et al. Molecular cloning and expression of Cro $\mathrm{s} 1$ : an occupational allergen from saffron pollen (Crocus sativus). Reports of biochemistry \& molecular biology. 2012;1(1):1-8.

16. Hosseinzadeh H, Nassiri-Asl M. Avicenna's (Ibn Sina) the Canon of Medicine and saffron (Crocus sativus): a review. Phytotherapy Res. 2013;27(4):475-83.

17. Marx W, Lane M, Rocks T, Ruusunen A, Loughman A, Lopresti A, et al. Effect of saffron supplementation on symptoms of depression and anxiety: a systematic review and meta-analysis. Nutr Rev. 2019.

18. Ghasemi T, Abnous K, Vahdati F, Mehri S, Razavi B, Hosseinzadeh H. Antidepressant effect of Crocus sativus aqueous extract and its effect on CREB, $B D N F$, and VGF transcript and protein levels in rat hippocampus. Drug research (Stuttg). 2015;65(7):337-43.

19. Li H, Shen Z, Lu Y, Lin F, Wu Y, Jiang Z. Muscle NT-3 levels increased by exercise training contribute to the improvement in caudal nerve conduction velocity in diabetic rats. Mol Med Rep. 2012;6(1):69-74.

20. Ying Z, Roy RR, Edgerton VR, Gomez-Pinilla F. Voluntary exercise increases neurotrophin-3 and its receptor TrkC in the spinal cord. Brain Res. 2003;987(1):93-99.

21. Dehghan F, Hajiaghaalipour F, Yusof A, Muniandy S, Hosseini SA, Heydari S, et al. Saffron with resistance exercise improves diabetic parameters through the GLUT4/AMPK pathway in-vitro and in-vivo. Sci Rep. 2016;6:25139.

22. Health NIo. Guide for the care and use of laboratory animals. National Academies. 1985.

23. Arqué G, Fotaki V, Fernández D, De Lagrán MM, Arbonés ML, Dierssen M. Impaired spatial learning strategies and novel object recognition in 
mice haploinsufficient for the dual specificity tyrosine-regulated kinase-1A (Dyrk1A). PLoS One. 2008;3(7):e2575.

24. Antunes M, Biala G. The novel object recognition memory: neurobiology, test procedure, and its modifications. Cogn Process. 2012;13(2):93-110.

25. Yang JL, Lin YT, Chuang PC, Bohr VA, Mattson MP. BDNF and exercise enhance neuronal DNA repair by stimulating CREBmediated production of apurinic/apyrimidinic endonuclease 1. Neuromolecular Med. 2014;16(1):161-174.

26. Marais L, Stein DJ, Daniels WM. Exercise increases $B D N F$ levels in the striatum and decreases depressive-like behavior in chronically stressed rats. Metab Brain Dis. 2009;24(4):587-97. 27. Vilela TC, Muller AP, Damiani AP, Macan TP, da Silva S, Canteiro PB, et al. Strength and Aerobic Exercises Improve Spatial Memory in Aging Rats Through Stimulating Distinct Neuroplasticity Mechanisms. Mol Neurobiol. 2017;54(10):7928-7937.

28. Hopkins ME, Nitecki R, Bucci DJ. Physical exercise during adolescence versus adulthood: differential effects on object recognition memory and brain-derived neurotrophic factor levels. Neuroscience. 2011;194:84-94.

29. Wang C, Cai X, Hu W, Li Z, Kong F, Chen X, et al. Investigation of the neuroprotective effects of crocin via antioxidant activities in HT22 cells and in mice with Alzheimer's disease. Int J Mol Med. 2019;43(2):956-966.
30. Tamaddonfard E, Farshid AA, Asri-Rezaee S, Javadi S, Khosravi V, Rahman B, et al. Crocin improved learning and memory impairments in streptozotocin-induced diabetic rats. Iran J Basic Med Sci. 2013;16(1):91-100.

31. Cassilhas RC, Tufik S, de Mello MT. Physical exercise, neuroplasticity, spatial learning and memory. Cell Mol Life Sci. 2016;73(5):975-83.

32. Hooshmand Moghadam B, Gaeini AA. Effect of complementary saffron and resistance training on serum levels of growth hormone, insulin-like growth factor-1 and testosterone in young men. Iranian J of endocrinology and metabolism. 2018; 20(4): 177-184.

33. Chen HT, Chung YC, Chen YJ, Ho SY, Wu HJ. Effects of Different Types of Exercise on Body Composition, Muscle Strength, and IGF-1 in the Elderly with Sarcopenic Obesity. J Am Geriatr Soc. 2017;65(4):827-832.

34. Zuzina AB, Vinarskaya AK, Balaban PM. Increase in serotonin precursor levels reinstates the context memory during reconsolidation. Invertebrate Neuroscience. 2019;19(3):8.

35. KARIMI GR, Hosseinzadeh H, KHALEGH PP. Study of antidepressant effect of aqueous and ethanolic extract of Crocus sativus in mice. 2001.

36. Sharma NK, Ryals JM, Gajewski BJ, Wright DE. Aerobic exercise alters analgesia and neurotrophin-3 synthesis in an animal model of chronic widespread pain. Phys Ther. 2010;90(5):714-725.

37. Akbari-Fakhrabadi M, Najafi M, Mortazavian S, Rasouli M, Memari AH, Shidfar F. Effect of saffron (Crocus sativus L.) and endurance training on mitochondrial biogenesis, endurance capacity, inflammation, antioxidant, and metabolic biomarkers in Wistar rats. J Food Biochem. 2019;43(8):e12946. 